\title{
Species Composition and Relative Abundance of Birds at Arbegona, Geremba Mountain Fragment Forest, Southern Ethiopia
}

\author{
Ziyad Jemal Husein ${ }^{1} \quad$ Mustefa Sultan Dalu ${ }^{2}$ \\ 1.Arsi Mountains National Park, Oromia Forest and Wildlife Enterprise, Asella, Ethiopia \\ 2.Department of Forestry, College of Agriculture and Environmental Science, Arsi University, Asella, Ethiopia
}

\begin{abstract}
Ethiopia is rich in biodiversity resources. Birds are one of the components of biodiversity. The status of birds in the present study areas is very little known. The objective of the study was to assess species composition and relative abundance of birds at geremba mountain fragment in southern Ethiopia. A stratified random sampling technique was employed. Accordingly, to collect data for the study, the whole habitat of the study area was each divided into dominant vegetation types. Consequently, Modified habitat, Alpine bamboo forest, and Sub-Afro alpine /Ericaceous belt were identified in geremba mountain fragment. A line transects count aided by binocular was employed to investigate avian species diversity, relative abundance and habitat association. Thus, 10 transect lines of $0.75 \mathrm{~km}$ with a width of $0.15 \mathrm{~km}$ or less was used to cover $30 \%$ of the area in geremba mountain fragment. Quantitative Biodiversity indices such as $\mathrm{H}^{\prime}, \mathrm{E}, \gamma$ and $\alpha$ to measure species richness were used to calculate the bird species diversity and two way- ANOVA was used to test for significant variation in species richness and abundance per season among habitats. A total of 74 bird species consisting of 5 near endemics, 2 globally threatened and 3 Palearctic migrants were recorded in geremba mountain fragment. Species richness and abundance varied between the three habitats in geremba mountain fragment. There was significant difference in species richness among habitats in geremba mountain fragment $(F 2,11=54.932 \mathrm{P}=0.000)$ at 0.001 significance level. In geremba mountain fragment the highest Shannon-Wiener diversity index $\left(\mathrm{H}^{\prime}=3.78\right)$ was recorded from the modified habitat while the lowest from the Sub Afro -alpine habitat $\left(\mathrm{H}^{\prime}=1.84\right)$. It can be concluded that the area has high species diversity good potential for bird watching tourism that can integrate economic gain with biodiversity conservation. Hence, urgent conservation measures and further detail research is recommended to conserve the bird species.
\end{abstract}

Keywords/Phrases: Avian species, Habitat types, Species richness, Species similarity

DOI: $10.7176 /$ ALST/71-01

\section{Introduction}

Ethiopia has diverse ecosystem ranging from humid forest and extensive wetlands in the west and southwest to the desert of Afar depression in the northeast. The country's flora and fauna are scattered from the highest mountain peaks over $4000 \mathrm{~m}$ asl to one of the lowest and hottest places on earth in Dallol depression, $116 \mathrm{~m}$ below sea level (Ethiopian Mapping Authority, 1988). This has helped to contribute for the existence of various forms of life in Ethiopia

Among 2100 bird species so far recorded in Africa (Sinclair and Ryan, 2003), Ethiopia harbors 926 species (Lepage, 2006) of which 21 species are endemic and 19 species are globally threatened (Redman et al., 2009). Out of the threated bird species of the country, two species are critically endangered, five species endangered, and 12 species vulnerable (Collar et al., 1994). In Ethiopia, 73 hot spots have been identified as Important Bird Areas (IBAs) and grouped into three conservation categories: 19 critical sites, 23 urgent sites and 31 highly sited areas (Mengistu, 2004).

Birds are one of the most important components of biodiversity with ecological, economic and esthetic values. Birds are known as efficient and cost-effective insect pest controllers, Fruit-eating birds help in dispersal of seeds and seeds may sprout wherever the droppings fall and certain birds like hummingbirds and sunbirds pollinate flowers that produce nectar. Birds through the ages have been the source of considerable fascination and folklore and have been used as symbols (Clout and Hay, 1989).

The distribution and abundance of many bird species are determined by the composition of the vegetation or habitat (Lee and Rotenberry, 2005). Climate influences habitats and movements of resident and migratory birds. Many species are characteristic of particular habitats or biomes (Metzger et al., 2009).

Studies from different parts of the world focused on characterizing the bird's species composition and abundance on specific regions of the country (eg. Study by Girma Mengesha and Afework Bekele, 2008). There are a few isolated reports of bird species diversity outside of protected areas in Ethiopia (Aerts et al., 2008), although there is better documentation of birds in protected areas (EWNHS, 1996). Comprehensive baseline information is lacking even for several of the endemic bird species. The status of birds in the present study areas is very little known. As a result, the present study is aimed to investigate bird species diversity, relative 
abundance and habitat association in geremba mountain fragment and contribute to the conservation of the species in the area.

\section{Materials and Methods \\ Study area}

Arbegona district is located between $6^{\circ} 38^{\prime}-6^{\circ} 49^{\prime} \mathrm{N}$ and $38^{\circ} 34^{\prime}-38^{\circ} 49^{\prime} \mathrm{E}$ (Fig.1). It is located $261 \mathrm{Km}$ South from Addis Ababa and $77 \mathrm{Km}$ South East from Hawassa town, the capital city of the zone. Geremba mountain fragment was the specific site where the present study was conducted.The area is found in Arbegona district in Sidama Zone of the Southern Nations, Nationalities, and Peoples' Regional state of Ethiopia (Fig.1).
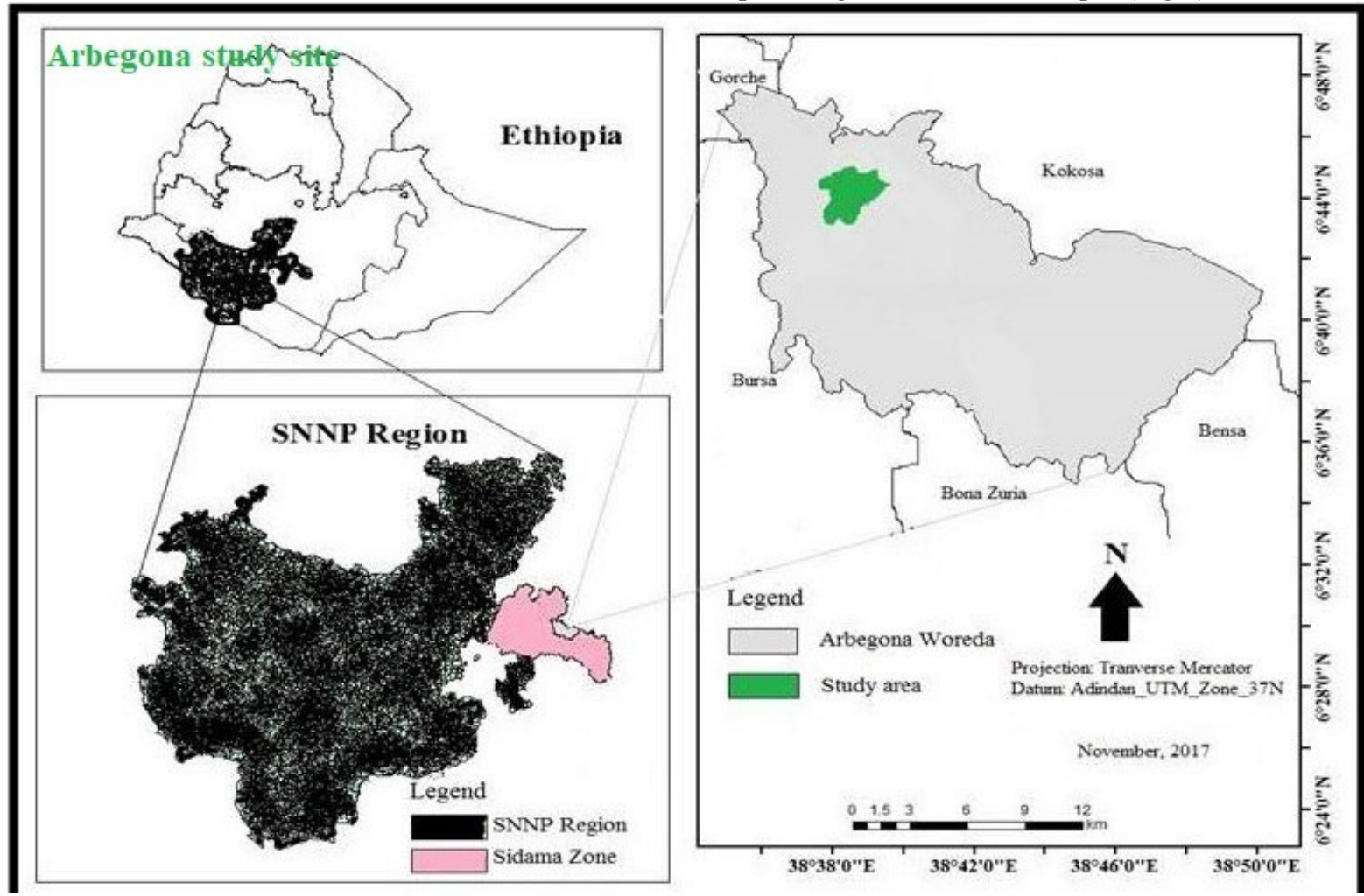

Figure 1: Location Map of Study Area

The district exhibit bimodal rainfall pattern, Arbegona district has a minor rainy season between the months of February to April and major rainfall between the months of July to October with an annual rain fall which ranges between 1250 to $1300 \mathrm{~mm}$, the temperature ranges between a minimum of $14 \mathrm{C}^{\circ}$ and a maximum of $18 \mathrm{C}^{\circ}$.

\section{Stratification of the study area and sampling design}

For this study, the Geremba mountain fragment was stratified into three habitat types [modified habitat at the lower, Alpine bamboo forest at the middle and Sub Afro-alpine habitat (Ericaceous belt)]at the higher altitude following vegetation type and altitudinal gradient. In Geremba mountain fragment, modified habitat represents areas with altitudes occurs from 3075-3165 $\mathrm{m}$ a.s.l. This habitat was disturbed habitat with livestock grazing and human encroachments.

The Alpine bamboo habitat represents valley and middle altitude areas between 3189-3229 $\mathrm{m}$ a.s.l., and was dominated by highland Bamboo (Arundinaria alpina).This habitat was relatively intact and undisturbed compared to other habitat types. The Sub Afro-alpine habitat (Ericaceous belt) habitat covers the upland areas with rugged topography (3291-3305 m a.s.l.) and little remnants of Erica arborea at the summit of mount garemba and intermixed with predominantly scattered stands of trees like Hypericum revoltum, Giant lobelia and Arundinaria alpina in Geremba Mountain fragment.

Based on the reconnaissance survey, sampling transects was systematically generated in a geographic information system (GIS) using ArcGIS software v. 10.1 (ESRI, 2012) in the Geremba mountain fragment. The total area of Garemba Mountain was $125,0000 \mathrm{~m}^{2}$ (1250 ha). Of these $30 \%$ ( $\left.375 \mathrm{ha}\right)$ of the area were sampled in Garemba Mountain. A stratified random sampling technique was employed in which transect placement was proportional to the area of the habitat types and represents each of the habitat types (Bibby et al., 1998; Lambert et al., 2009;Shimelis and Afework, 2008). Accordingly, a total of 10 transect lines, (Five (5) transects in modified habitat, four (4) transects in alpine bamboo forest and one (1) transect conducted in Sub Afro-alpine 
(ericaceous belt) habitat in Garemba Mountain fragment (Fig.2). The distance between two adjacent transects was $0.25 \mathrm{~km}$ in Geremba mountain fragment. The length of each transect line was $0.75 \mathrm{~km}$ with a width of $0.15 \mathrm{~km}$ or less in Garemba mountain fragment. To avoid edge effect, transect lines were spaced $250 \mathrm{~m}$ in Garemba Mountain fragment from the road side (edge of the forest). Line transect method was used since the study area is accessible and species can be detected along transect line. With line transect method it is possible to cover large areas and can generate more species richness efficiently (Bibby et al., 1992).

Therefore, this method is very important since comprehensive baseline information and status of bird species in Garemba Mountain fragment is lacking.

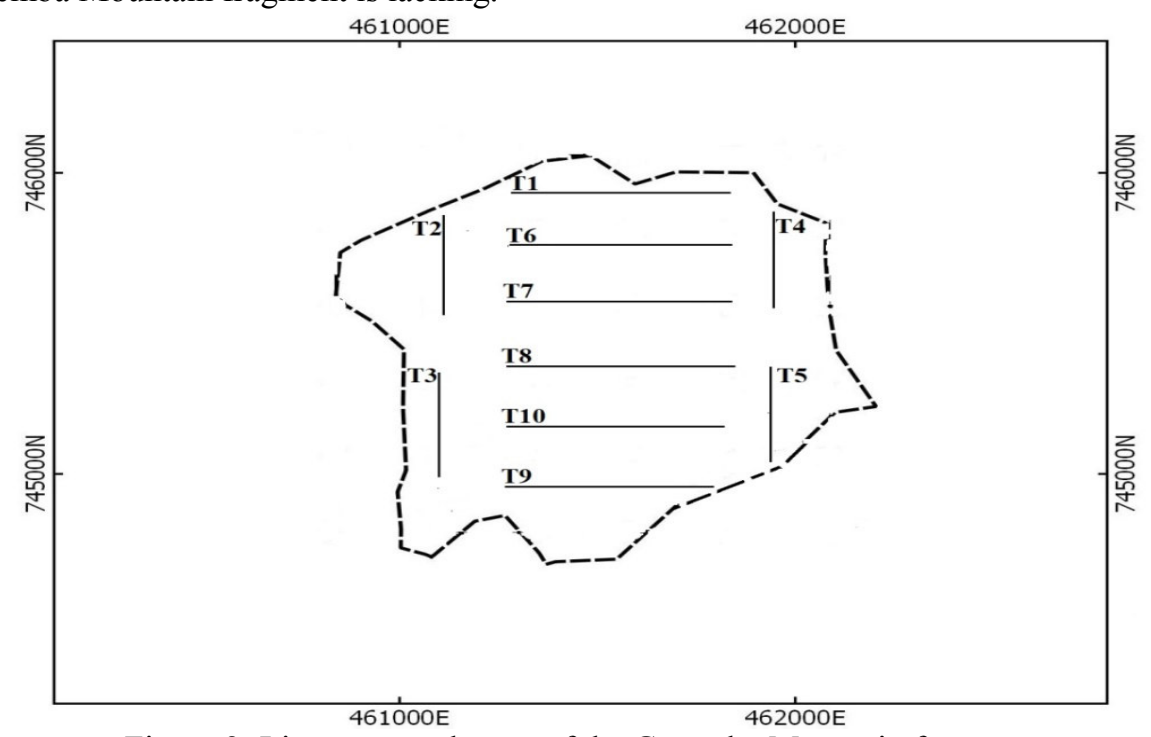

Figure 2: Line transect layout of the Garemba Mountain fragment.

\section{Data collection and analysis}

Data collection was carried out on foot walking along transect lines. Bird identifications and counting of individuals conducted by direct observations aided with naked eye and binoculars (10x50). Sound records and photography were also taken for further confirmation by using Digital Camera. Location and distance of the observed birds was determined and recorded along transect lines using Geographic positioning system (GPS). Each day of survey, arrive at the starting point approximately 20 minutes before sunrise so that counting can begin at sunrise to minimize the effect of time and weather conditions on bird detectability. Birds were counted when they were active in the mornings from 06:30-10:00 $\mathrm{h}$ and in the afternoon from 15:30-18.00 $\mathrm{h}$ (Daviosdottir, 2010). Unfavorable weather (strong wind or rain) was also being considered.

A bird flying over the area was observed and recorded on data gathering worksheets to identify for species richness. For identification of species, plumage pattern, size, shape, color, songs and calls were considered as important parameters (Afework and Shimelis, 2009). Songs and calls were also used for identifying nocturnal species.

Avian diversity of each habitat analyzed using Shannon-Wiener diversity Index (H') (Shannon and Wiener, 1949). Quantitative indices to measure species richness was used as $\alpha$-diversity the average species richness per transect within a given habitat and $\gamma$-diversity as the habitat level richness (Schmitt et al., 2010).

Percent relative abundance was calculated using formula $(\%)=n / N \times 100$ where, $n$ is the number of individuals of particular species recorded and $\mathrm{N}$ is the total number of individuals of the species. Data obtained during the survey was analyzed by using two-way ANOVA to analyze the effect of season and habitat on bird species richness and abundance.

\section{Results}

\section{Species Composition and Relative Abundance}

A total of 618 individuals of birds grouped into 12 orders, 35 families and 74 species were recorded from Geremba mountain fragment (Table 3). Among the recorded species, Wattled ibis (Bostrychia carunculata), Thick billed raven (Corvus crassirostris), Alpine chat (Cercomela sordida), Black winged love bird (Agapornis taranta) and Rouget's Rail (Rougetius rougetii) were endemic to Ethiopia and Eritrea in Geremba mountain fragment. Two globally threatened bird species i.e Hooded vulture (Psophocichla litsipsirupa) was endangered and Rougets rail (Onychognathus morio) was near threatened bird species (IUCN red list, 2016) (Table 3).

Among the recorded bird species 71 were resident and 3 were Palearctic migrants in Geremba mountain fragment (Table 3). The order Passeriformes accounted, the highest number of families consisting 21 families 
and 43 species recorded in the Arebgona followed by the family Accipitridae with 9 species recorded and Sylviidae which had 5 species record (Table 3).

Table 3: Bird species recorded at Geremba mountain fragment (a, Near Endemic c, endangered b, Endemic e, near threatened NM, Northern Migratory AM, Inter-African migrant)

\begin{tabular}{|c|c|c|c|c|c|c|c|c|}
\hline \multirow[t]{2}{*}{ Order } & \multirow[t]{2}{*}{ Family } & \multirow[t]{2}{*}{ Common name } & \multirow[t]{2}{*}{ Scientific Name } & \multicolumn{3}{|c|}{ Abundance } & \multirow[b]{2}{*}{$\begin{array}{l}\text { RA } \\
\text { (\%) }\end{array}$} & \multirow[b]{2}{*}{ Rank } \\
\hline & & & & Wet & Dry & Total & & \\
\hline Passeriformes & Estrildidae & Yellow bellied waxbill & Coccopygia quartinia & 17 & 22 & 39 & 6.31 & $1^{\text {st }}$ \\
\hline Passeriformes & Turdidae & Alpine chat & Cercomela sordida & 20 & 19 & 39 & 6.31 & $1^{\text {st }}$ \\
\hline Passeriformes & Rallidae & Rupels robin chat & Cossypha semirufa & 20 & 15 & 35 & 5.66 & $3^{\text {rd }}$ \\
\hline Passeriformes & Zosteropidae & Mountain thrush & Turdus olivaceus & 16 & 15 & 31 & 5.02 & $4^{\text {th }}$ \\
\hline Passeriformes & Nectariniidae & Wattled Ibis & Cinnyris venustus ${ }^{\mathrm{a}}$ & 14 & 12 & 26 & 4.21 & $5^{\text {th }}$ \\
\hline Columbiformes & Columbidae & Streaky seed eater & Columba guinea & 17 & 9 & 26 & 4.21 & $5^{\text {th }}$ \\
\hline Passeriformes & Passeridae & Takazze sun bird & Passer swainsonii & 17 & 6 & 23 & 3.72 & $7^{\text {th }}$ \\
\hline Passeriformes & Buphagidae & Red winged starling & Buphagus erythrorhynchus & 15 & 7 & 22 & 3.56 & $8^{\text {th }}$ \\
\hline Passeriformes & Sylviidae & Cinnamon bracken warbler & Batis minor & 11 & 8 & 19 & 3.07 & $9^{\text {th }}$ \\
\hline Passeriformes & Alaudidae & Thick billed raven & Galerida theklae $\mathbf{a}^{\mathbf{a}}$ & 10 & 7 & 17 & 2.75 & $10^{\text {th }}$ \\
\hline Passeriformes & Nectariniidae & Montane white eye & Nectarinia famosa & 6 & 11 & 17 & 2.75 & $10^{\text {th }}$ \\
\hline Passeriformes & Ploceidae & Baglafecht weaver & Ploceus baglafecht & 11 & 5 & 16 & 2.59 & $12^{\text {th }}$ \\
\hline Passeriformes & Sylviidae & Brown rumped seed eater & Parisoma lugens & 10 & 6 & 16 & 2.59 & $12^{\text {th }}$ \\
\hline Falconiformes & Accipitridae & Tawny flanked prinia & Aquila rapax & 8 & 5 & 13 & 2.10 & $14^{\text {th }}$ \\
\hline Galliformes & Phasianidae & Chestnut naped francolin & Bubo capensis & 7 & 6 & 13 & 2.10 & $14^{\text {th }}$ \\
\hline Passeriformes & Corvidae & Variable sun bird & Corvus crassirostris & 10 & 2 & 12 & 1.94 & $16^{\text {th }}$ \\
\hline Passeriformes & Fringillidae & Bush petronia & Crithagra tristriatus & 7 & 4 & 11 & 1.78 & $17^{\text {th }}$ \\
\hline Galliformes & Phasianidae & Chinspot batis & Pternistis castaneicollis & 11 & 0 & 11 & 1.78 & $17^{\text {th }}$ \\
\hline Coraciiformes & Bucerotidae & Dusky turtle dove & Tockus alboterminatus & 2 & 9 & 11 & 1.78 & $17^{\text {th }}$ \\
\hline Passeriformes & Cisticolidae & Green backed eremomela & Camaroptera brachyura & 8 & 2 & 10 & 1.62 & $20^{\text {th }}$ \\
\hline Passeriformes & Motacillidae & Yellow breasted apalis & Motacilla flavida & 6 & 3 & 9 & 1.46 & $21^{\text {th }}$ \\
\hline Passeriformes & Sylviidae & Ground scraper thrush & Eremomela canescens & 5 & 4 & 9 & 1.46 & $21^{\text {th }}$ \\
\hline Passeriformes & Accipitridae & Malachite sun bird & Gypaetus barbatus & 5 & 4 & 9 & 1.46 & $21^{\text {th }}$ \\
\hline Passeriformes & Paridae & White backed black tit & Parus leuconotus & 5 & 4 & 9 & 1.46 & $21^{\text {th }}$ \\
\hline Passeriformes & Muscicapidae & Abyssinian slaty fly catcher & Melaenornis chocolatina ${ }^{\mathbf{a}}$ & 4 & 3 & 7 & 1.13 & $25^{\text {th }}$ \\
\hline Passeriformes & Muscicapidae & Scaly francolin & Cossypha semirufa & 3 & 4 & 7 & 1.13 & $25^{\text {th }}$ \\
\hline Columbiformes & Columbidae & African olive pegion & Columba arquatrix & 0 & 7 & 7 & 1.13 & $25^{\text {th }}$ \\
\hline Piciformes & Indicatoridae & Green backed cameroptera & Indicator indicator & 5 & 1 & 6 & 0.97 & $28^{\text {th }}$ \\
\hline Passeriformes & Monarchidae & African dusk flycatcher & Muscicapa adusta & 4 & 2 & 6 & 0.97 & $28^{\text {th }}$ \\
\hline Passeriformes & Fringillidae & Swaisons sparrow weaver & Serinus striolatus & 3 & 3 & 6 & 0.97 & $28^{\text {th }}$ \\
\hline Passeriformes & Turdidae & Abyssinian ground thrush & Zoothera piaggiae & 2 & 4 & 6 & 0.97 & $28^{\text {th }}$ \\
\hline Passeriformes & Passeridae & Cape crow & Petronia dentata & 2 & 4 & 6 & 0.97 & $28^{\text {th }}$ \\
\hline Passeriformes & Pipridae & Barn swallow & Manacus manacus & 4 & 1 & 5 & 0.81 & $33^{\text {th }}$ \\
\hline Passeriformes & Muscicapidae & Tawny eagle & Nectarinia tacazze & 3 & 2 & 5 & 0.81 & $33^{\text {th }}$ \\
\hline Passeriformes & Fringillidae & African citril & Serinus citrinelloides & 2 & 3 & 5 & 0.81 & $33^{\text {th }}$ \\
\hline Falconiformes & Accipitridae & Augur buzzard & Buteo augur & 3 & 2 & 5 & 0.81 & $33^{\text {th }}$ \\
\hline Columbiformes & Columbidae & Fantailed raven & Streptope lialugens & 4 & 1 & 5 & 0.81 & $33^{\text {th }}$ \\
\hline Coliiformes & Collidae & Speckled pegion & Colius striatus & 3 & 2 & 5 & 0.81 & $33^{\text {th }}$ \\
\hline Coliiformes & Apodidae & African black swift & Apus parvus & 5 & 0 & 5 & 0.81 & $33^{\text {th }}$ \\
\hline Passeriformes & Cisticolidae & Yellow bellied eremomela & Eremomela icteropygialis & 5 & 0 & 5 & 0.81 & $33^{\text {th }}$ \\
\hline Passeriformes & Estrildidae & Common waxbill & phylloscopus collybita & 3 & 2 & 5 & 0.81 & $33^{\text {th }}$ \\
\hline Passeriformes & Nectariniidae & Pallid harrier & Cyanomitraolivacea & 1 & 3 & 4 & 0.65 & $42^{\text {th }}$ \\
\hline Passeriformes & Sylviidae & Willow warbler & Phylloscopus trochilus & 1 & 3 & 4 & 0.65 & $42^{\text {th }}$ \\
\hline Accipitriformes & Accipitridae & Pied crow & Circus macrourus & 2 & 2 & 4 & 0.65 & $42^{\text {th }}$ \\
\hline Piciformes & Accipitridae & Greater honey guide & Accipiter melanoleucus & 2 & 2 & 4 & 0.65 & $42^{\text {th }}$ \\
\hline Falconiformes & Emberizidae & Lammargier & Emberiza striolata & 2 & 2 & 4 & 0.65 & $42^{\text {th }}$ \\
\hline Passeriformes & Turdidae & Olive sun bird & Turdus olivaceus & 3 & 0 & 3 & 0.49 & $48^{\text {th }}$ \\
\hline Passeriformes & Muscicapidae & Speckled mouse bird & Melaenornis pammelania & 2 & 1 & 3 & 0.49 & $48^{\text {th }}$ \\
\hline Passeriformes & Motacillidae & Yellow wagtail $^{\text {NM }}$ & Motacilla flava & 1 & 2 & 3 & 0.49 & $48^{\text {th }}$ \\
\hline & & *African paradise & & & & & & \\
\hline Passeriformes & Monarchidae & flycatcher ${ }^{\mathrm{AM}}$ & Terpsiphone viridis & 0 & 3 & 3 & 0.49 & $48^{\text {th }}$ \\
\hline Passeriformes & Turdidae & Hooded volture & Psophocichla litsipsirupa ${ }^{\mathbf{c}}$ & 0 & 5 & 3 & 0.49 & $48^{\text {th }}$ \\
\hline Falconiformes & Accipitridae & House bunting & Necrosyrtes monachus & 3 & 0 & 3 & 0.49 & $48^{\text {th }}$ \\
\hline Psittaciformes & Sylviidae & Collared sun bird & Bradypterus cinnamomeus & 3 & 0 & 3 & 0.49 & $48^{\text {th }}$ \\
\hline Piciformes & Indicatoridae & Southern black fly catcher & Indicator variegatus & 2 & 0 & 2 & 0.32 & $55^{\text {th }}$ \\
\hline Pelecaniformes & Threskiornithidae & White and black manninkin & Bostrychia carunculata & 2 & 0 & 2 & 0.32 & $55^{\text {th }}$ \\
\hline Passeriformes & Hirundinidae & Black chested snake eagle & Hirundo rustica & 2 & 0 & 2 & 0.32 & $55^{\text {th }}$ \\
\hline Passeriformes & Nectariniidae & Common bulbul & Hedydipna collaris & 2 & 0 & 2 & 0.32 & $55^{\text {th }}$ \\
\hline Passeriformes & 'Pycnonotidae & Common chifchaff & Pycnonotus barbatus & 2 & 0 & 2 & 0.32 & $55^{\text {th }}$ \\
\hline Passeriformes & 'Sturnidae & Rougets rail & Onychognathus morio ${ }^{\mathbf{a e}}$ & 2 & 0 & 2 & 0.32 & $55^{\text {th }}$ \\
\hline Passeriformes & Cisticolidae & Thekla lark & Prinia subflava & 2 & 0 & 2 & 0.32 & $55^{\text {th }}$ \\
\hline Passeriformes & Estrildidae & Crowned hornbill & Estrilda astrild & 1 & 1 & 2 & 0.32 & $55^{\text {th }}$ \\
\hline Galliformes & Phasianidae & Scaly throated honey guide & Pternistis squamatus & 2 & 0 & 2 & 0.32 & $55^{\text {th }}$ \\
\hline Psittaciformes & Accipitridae & Black winged Love bird & Milvus migrans ${ }^{\mathrm{a}}$ & 2 & 0 & 2 & 0.32 & $55^{\text {th }}$ \\
\hline Passeriformes & Corvidae & Red billed oxpecker & Corvus albus & 2 & 0 & 2 & 0.32 & $55^{\text {th }}$ \\
\hline Psittaciformes & Psittaculidae & Blue headed coucal & Agapornis taranta & 2 & 0 & 2 & 0.32 & $55^{\text {th }}$ \\
\hline Psittaciformes & Corvidae & Cape eagle owl & Corvus capensis & 1 & 1 & 2 & 0.32 & $55^{\text {th }}$ \\
\hline Passeriformes & Accipitridae & Black kite & Circaetus pectoralis & 0 & 2 & 2 & 0.32 & $55^{\text {th }}$ \\
\hline Passeriformes & Corvidae & Great sparrow hawk & Corvus rhipidurus & 1 & 0 & 1 & 0.16 & $69^{\text {th }}$ \\
\hline Cuculiformes & Cuculidae & Brown parisoma & Centropus monachus & 1 & 0 & 1 & 0.16 & $69^{\text {th }}$ \\
\hline Apodiformes & Apodidae & $*$ Alpine swift ${ }^{\mathrm{NM}}$ & Tachymarptis melba & 1 & 0 & 1 & 0.16 & $69^{\text {th }}$ \\
\hline Accipitriformes & Accipitridae & Yellow billed kite & Milvus aegyptius & 1 & 0 & 1 & 0.16 & $69^{\text {th }}$ \\
\hline Accipitriformes & Timaliidae & Abyssinian cat bird & Parophasma galinier $^{\mathbf{b}}$ & 1 & 0 & 1 & 0.16 & $69^{\text {th }}$ \\
\hline Falconiformes & Falconidae & African hobby & Falco cuvierii & 1 & 0 & 1 & 0.16 & $69^{\text {th }}$ \\
\hline
\end{tabular}

Based on the percent relative abundance computation, Alpine chat (Cercomela sordida) $(6.31 \%)$ was the 
most abundant bird species when all habitat types considered together in Geremba mountain fragment (Table 3). When each habitat type was considered separately, in decreasing order Alpine chat (Cercomela sordida) $(6.31 \%)$, Rupels robin chat (Cossypha semirufa) (21.21\%) and Chinspot batis (Castanei collis) (7.31\%) were the most abundant species in the modified habitat, Sub Afro-alpine, and Alpine bamboo forest respectively in Geremba mountain fragment(Table 4).

Table 4: Top five ranking abundant species among three habitat types and across the study area based on percent relative abundance in Geremba mountain fragment

\begin{tabular}{|c|c|c|c|c|c|c|c|c|}
\hline \multirow[b]{3}{*}{ Species Name } & \multicolumn{6}{|c|}{ Habitat Types } & & \\
\hline & \multicolumn{2}{|c|}{$\begin{array}{c}\text { Sub } \\
\text { Afro-alpine }\end{array}$} & \multicolumn{2}{|c|}{$\begin{array}{c}\text { Alpine } \\
\text { bamb } \underline{\text { bago }} \text { forest }\end{array}$} & \multicolumn{2}{|c|}{ Modifified } & \multicolumn{2}{|c|}{ Across } \\
\hline & RA $(\%)$ & Rank & $\mathrm{RA}(\%)$ & Rank & RA $(\%)$ & Rank & $\mathrm{RA}(\%)$ & Rank \\
\hline Alpine chat & - & - & - & - & 10.46 & $1^{\text {st }}$ & 6.31 & $1^{\text {st }}$ \\
\hline Yellow bellied waxbill & - & - & - & - & 9.38 & $2^{\text {nd }}$ & 6.31 & $1^{\text {st }}$ \\
\hline Rupels robin chat & 21.21 & $1^{\text {st }}$ & 4.91 & $5^{\text {th }}$ & 4.96 & $5^{\text {th }}$ & 5.66 & $3^{\text {rd }}$ \\
\hline Mountain thrush & - & - & - & - & 6.17 & $3^{\text {rd }}$ & 5.02 & $4^{\text {th }}$ \\
\hline Streaky seed eater & - & - & - & - & 6.17 & $4^{\text {th }}$ & 4.21 & $5^{\text {th }}$ \\
\hline Takazze sun bird & 7.58 & $5^{\text {th }}$ & - & - & - & - & 3.72 & $6^{\text {th }}$ \\
\hline Cinnamon bracken warbler & 12.12 & $2^{\text {nd }}$ & 5.61 & $3^{\text {rd }}$ & - & - & 3.07 & $7^{\text {th }}$ \\
\hline Thick billed raven & 10.61 & $4^{\text {th }}$ & - & - & - & - & 2.91 & $8^{\text {th }}$ \\
\hline Montane white eye & - & - & 4.91 & $4^{\text {th }}$ & - & - & 2.75 & $9^{\text {th }}$ \\
\hline Tawny flanked prinia & 10.61 & $3^{\text {rd }}$ & - & - & - & - & 2.10 & $10^{\text {th }}$ \\
\hline Bush petronia & - & - & 7.02 & $2^{\text {nd }}$ & - & - & 1.78 & $11^{\text {th }}$ \\
\hline Chinspot batis & - & - & 7.37 & $1^{\text {st }}$ & - & - & 1.78 & $11^{\text {th }}$ \\
\hline
\end{tabular}

\section{Diversity}

In Geremba mountain fragment variation in the number of bird species was observed among the habitats and between seasons in the same habitat. In Geremba mountain fragment the highest mean number of species per

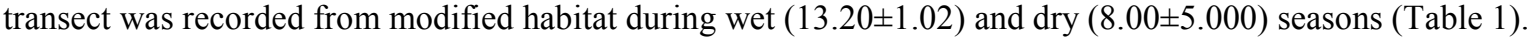

The highest variation in mean abundance per transect between dry and wet seasons was recorded in the modified habitat while Sub Afro-alpine (Ericaceous belt) had the least mean number of species richness in wet season (4.25 \pm 0.48$)$ and dry season $(3.00 \pm 0.41)$ (Table 1$)$. The evidence from the present study also shows variations were also observed in species diversity among the different habitat types during the wet and dry seasons in Geremba mountain fragment. The modified habitat supported the highest avian diversity during wet $\left(H^{\prime}=3.710\right)$ and dry $\left(H^{\prime}=3.281\right)$ seasons in Geremba mountain fragment (Table 1). In Geremba mountain fragment, Sub Afro- alpine (Ericaceous belt) supported the least diversity of avian during the wet $\left(\mathrm{H}^{\prime}=2.272\right.$ ) and dry $\left(\mathrm{H}^{\prime}=1.840\right)$ seasons (Table 1$)$.

There was also seasonal variation of species evenness with in habitat between seasons Sub Afro-alpine (Ericaceous belt) accounted species evenness during wet $(E=0.947)$ and dry $(E=0.946)$ seasons in Geremba mountain fragment. However the even distribution that were recorded in modified habitat during wet $(0.885)$ and dry (0.895) seasons in Geremba

Mountain fragment (Table1).

Table 1: Seasonal variation in bird diversity in Geremba mountain fragment among the different habitat types

\begin{tabular}{|c|c|c|c|c|c|c|c|c|c|}
\hline \multirow[b]{2}{*}{ Study areas } & \multirow[b]{2}{*}{ Habitat types } & \multicolumn{8}{|c|}{ Diversity Measures } \\
\hline & & Season & $\begin{array}{l}\text { BSRPH }(\gamma- \\
\text { diversity) }\end{array}$ & $\begin{array}{c}\text { BMSRPT } \\
(\alpha \text {-diversity) }\end{array}$ & BSAPH & BMSAPT & $\mathrm{H}^{\prime}$ & $\mathrm{H}^{\prime} \max$ & Evenness \\
\hline & $\begin{array}{l}\text { Alpine } \\
\text { bamboo }\end{array}$ & dry & 21.000 & $5.25 \pm 2.95$ & 116.000 & $22.67 \pm 11.465$ & 2.817 & 3.045 & 0.925 \\
\hline & forest & wet & 32.000 & $7.80 \pm 2.27$ & 197.000 & $23.83 \pm 4.103$ & 3.219 & 3.466 & 0.929 \\
\hline & & dry & 39.000 & $8.00 \pm 5.00$ & 319.000 & $31 \pm 8.000$ & 3.281 & 3.664 & 0.895 \\
\hline Geremba & Modified & wet & 66.000 & $13.20 \pm 1.02$ & 495.000 & $39 \pm 2.437$ & 3.710 & 4.190 & 0.885 \\
\hline mountain & Sub Afro & dry & 7.000 & $3.00 \pm 0.41$ & 17.000 & $17.33 \pm 9.262$ & 1.840 & 1.946 & 0.946 \\
\hline fragment & alpine & wet & 11.000 & $4.25 \pm 0.48$ & 49.000 & $19.67 \pm 7.535$ & 2.272 & 2.398 & 0.947 \\
\hline
\end{tabular}

BSRPH: Bird species richness per habitat

BMSRPT: Bird mean species richness per transect

BSAPH: Bird species abundance per habitat

BMSAPT: Bird mean species abundance per transect 


\section{Discussion}

Diversity

In Geremba mountain fragment the modified habitat had the most diversified avian species and most evenness as compared to the other habitat types. This variation could be due to variation in habitat heterogeneity. The more heterogeneous vegetation and vegetation strata in the modified habitat compared to the other habitat types could have provided several niches for birds making the modified habitat with high diversity of birds. The importance of the availability of different vegetation strata for different bird species is also supported by other studies. For example, Cueto and Casenava (1999) reported positive correlation between bird species richness and the availability of vegetation strata. Similarly, Erdelen (1984) indicated significant correlation between bird species diversity and vegetation structure.

The highest number of species recorded from modified habitat during wet and dry seasons. The outcome of this result coincides with the report of Karr (1976); Janzen (1967) and Smythe (1970) where structurally complex vegetation buffers the influence of seasonality and there is a great stability in resource availability, which allows species to occur as residents throughout the year. Species richness of different feeding guilds might respond differently to changes in vegetation structure and complexity across tropical ecosystems (Oliveira-Filho et al., 2013). During the wet season, flowering plants were flourishing and as a result, food was plentiful for birds in almost all the habitats. However, during the dry season, the deciduous trees defoliate and in the absence of food, many species of birds were restricted to specific habitat where sufficient resource was available.

The variations in species composition recorded during the wet and dry seasons among habitats were significantly different. This is also reported by Aynalem and Afework (2008) who showed the important effect of season or the role of climate in affecting the diversity of birds of a particular habitat. According to Karr (1976), the distinct seasonality of rainfall and seasonal variation in the abundance of food resources result in seasonal changes in the abundance of birds.

The lowest species richness from the Sub Afro-alpine habitat in Geremba mountain fragment was mainly attributed due to altitudinal, light, edaphic factors and climatic factor (Ahmed, 2013).

\section{Relative Abundance}

The relative abundance of bird species during wet and dry seasons might be related to the availability of food, habitat condition and breeding season of the species. Therefore, species distribution and abundance can be influenced by seasonal variation. Many factors could account for this. For example, Karr (1976) related the seasonality in the number of bird species with the availability of resources such as food and vegetation strata and found that the number of bird species varied seasonally with peaks in the late dry and early wet seasons.

In Geremba mountain fragment in terms of percent relative abundance both Yellow bellied waxbill (Coccopygia quartinia) and Alpine chat (Cercomela sordida) had the highest relative abundance in modified habitat. The relative abundance of birds in the study area is related to the availability of food, habitat condition and breeding season of the species. Similar result was also obtained by Girma and Afework (2008) who reported positive correlation between bird species richness and the availability of vegetation strata. Similarly, Chace et al. (2006) reported that birds respond to changes in vegetation composition and structure, which in turn affects their food resources.

\section{Acknowledgement}

We appreciate Wondo Genet College of Forestry and Natural Resources, Hawassa University for funding this research.

\section{References}

Aerts, R., F. Lerouge, E. Novmber, L. Lens, M. Hermay and B. Muys. 2008. Land rehabilitation and the conservation of birds in a degraded Afromontane landscape in northern Ethiopia.Biodiversity and Conservation 17: 53-69.

Afework Bekele and Shimelis Aynalem. 2009. Species composition, relative abundance and habitat association of the bird fauna of the montane forest of Zegie Peninsula and nearby Islands, Lake Tana, Ethiopia. SINET: Ethiopian Journal of Science 32:45-56.

Ahmed, A.A. 2013. Diversity and distribution of the Afro-alpine flora of Eastern Africa with special reference to the taxonomy of the genus Pentaschistis (poaceae).PhD Thesis, Kenyatta University, Nairobi. 258p.

Bibby, C. J., Burgess, N. D and D. Hills. 1992. Birds census technique. Tokyo, Toronto. Academic press 30: 8696.

Bibby, C.J., S. Marsden, and M. Jones. 1998. Bird surveys. Expedition Advisory Centre.

Chace, J.F., and J. Walsh. 2006. Urban effects on native avifauna: A review. Landscape Urban Plan 74:46-69.

Clout, M.N. and J.R. Hay. 1989. The importance of birds as browsers, pollinators and seed dispersers in New Zealand forest.New Zealand journal of ecology.27-33. 
Cueto, V.R. and J.L. Casenava. 1999. Determinants of bird species richness. Role of climate and vegetation structure at regional scale. Journal of Biogeography 26: 487-492.

Erdelen, M. 1984. Bird communities and vegetation structure: I.Correlations and comparison of simple and diversity indices. Oecologia 61: 277-284.

EWNHS. 1996. Important Bird Areas of Ethiopia. Ethiopian Wildlife Conservation Organization, Addis Ababa, Ethiopia 454p.

Girma Mengesha and Afework Bekele. 2008. Diversity and relative abundance of birds of Alatish National Park. International Journal of Ecology and Environmental Sciences 34:215-222.

Karr, J.R. 1976. Seasonality resource availability and community diversity in tropical bird communities. The American Naturalist 110: 937-974.

Lee, P.Y. and J.T. Rotenberry. 2005. Relationships between bird species and tree species assemblages in forested habitats of north eastern America. Journal of Biogeography 32:1139-1150.

Lepage, D. (2011). Avibase. Bird checklists of the world. Birdlife International. Downloaded from http://www.bsceoc.org/avibase/

Mengistu S, Getahun A Eds. 2004. The Wildlife Conservation Areas of Ethiopia: Current Status and Future Prospect . Prospect. Biological Society of Ethiopia, Addis Ababa. pp. 1-5.

Metzger, J. P., A. C. Martensen, M. Dixo, L. C. Bernacci. M. C.Ribeiro, A. M. G. Teixeira and R. Pardini. 2009. Time-lag in biological responses to landscape changes in a highly dynamic Atlantic forest region. Biological Conservation 142: 1166-1177.

Oliveira-Filho, A. T. , J. C. Budke, J. A. Jarenkow, P. V. Eisenlohr, and D. R. M. Neves. 2013. Delving into the variations in tree species composition and richness across South American subtropical Atlantic and Pampean forest. Journal of Plant Ecology 8: 242-260.

Redman, N., Stevenson. T. and Fanashawe. J. (2009). Birds of the Horn of Africa. Princeton

Schmitt, C.B., M. Denich, S. Demissew, I. Friis, and H.J. Boehmer, 2010. Floristic diversity in fragmented Afromontane rainforest: Altitudinal variation and conservation importance. Applied Vegetation Science 13: 291-30.University Press, Princeton and Oxford, 496 pp.

Weldemariam Tesfahunegny Bezabh. 2006. A guide to a complete annotated checklist of the birds of Ethiopia. 